\title{
Pancreaticoduodenal artery aneurysm: Treatment outcomes of a rare disorder
}

\author{
Hüseyin Onur Aydın ${ }^{1 *}$, Ebru Hatice Ayvazoğlu Soy ${ }^{1}$, atih Boyvat $^{2}$, Sedat Yıldırım ${ }^{1}$, Mehmet Haberal ${ }^{1}$
}

\begin{abstract}
Objective: Pancreaticoduodenal artery aneurysm is a rare vascular disorder that manifests with a clinical presentation ranging from asymptomatic cases to hemorrhagic shock due to aneurysm rupture. In this study, we aimed to evaluate the outcomes of patients treated with different methods for pancreaticoduodenal artery aneurysm.
\end{abstract}

Material and Methods: A total of 5 patients who were treated and followed for pancreaticoduodenal artery aneurysm between January 2011 and January 2018 were enrolled in the study. Standard endovascular embolization and surgical resection were performed. The demographic findings and treatment outcomes were compared.

Results: All patients were shown to have a pancreaticoduodenal artery aneurysm by radiological methods. The most common complaint was abdominal pain. The mean aneurysm size was $2.8 \mathrm{~cm}$. Two patients had coeliac axis stenosis and occlusion, one had hypertension, one had Marfan syndrome, and one had chronic pancreatitis as the possible etiological cause. One patient with occlusion of the coeliac axis had thrombus, one patient with coeliac axis stenosis had median arcuate ligament compression. Three patients were treated with embolization, one with surgery, and one with low-molecular weight heparin.

Conclusion: Different etiological factors and multiple vascular problems accompanying the disorder create difficulties for establishing a treatment algorithm. Due to the rare nature of the disease, studies available in the literature involve limited numbers of patients, as was also the case in our study. There appeared to be no significant differences between the treatment and follow-up duration among the different treatment methods we applied for our patients.

Key words: pancreaticoduodenal artery aneurysm, endovascular treatment, surgical treatment

\section{Introduction}

Pancreaticoduodenal artery (PDA) aneurysm is a rare condition. Its incidence has been reported as $2 \%$ of all splanchnic area aneurysms. Pseudo whoaneurysms may occur as a result of trauma, congenital malformations, pancreatitis, collagen tissue disorders, and systemic vasculitis (1). It is considered that all true aneurysms develop from coeliac axis stenosis (2). Seventy percent of these patients have coeliac axis stenosis as a result of median arcuate ligament compression (3). Moreover, stenosis of common hepatic artery may accompany this condition (4). It is considered that PDA aneurysms develop due to increased retrograde blood flow in the pancreaticoduodenal arcuate as a result of coeliac axis or hepatic artery stenosis. Today, with advances in imaging methods, the number of diagnosed PDA aneurysms has seen an increase.
While asymptomatic cases are incidentally diagnosed, the diagnosis may also be made as a result of chronic abdominal pain, chronic intestinal angina, gastrointestinal bleeding, and acute abdomen. In more than half of reported cases there may exist hemorrhagic shock secondary to aneurysm rupture (5). There is no established definitive treatment algorithm and different treatment methods are recommended, including endovascular interventions and surgical procedures.

The rare nature of the disease results in available studies in the literature being in the form of case reports or reports of small patient groups. Therefore, new studies are needed on possible etiological factors and treatment modalities. Our study aimed to compare etiological factors and to assess the outcomes of patients treated with different treatment methods for PDA aneurysms. 


\section{Material and Methods}

Five patients who treated for PDA aneurysm between January 2011 and June 2018 were included in the study. This study was approved by Baskent University Institutional Review Board (Project no: KA 18/250) and supported by Baskent University Research Fund. Each patient signed an approved informed consent before the treatment. The retrospective analysis of personal health data of study patients was in accordance with the Declaration of Helsinki. Patients' age, sex, symptoms at diagnosis, size of aneurysm, presence or absence of coeliac axis stenosis and median arcuate ligament compression, type of treatment, and result of the treatments were evaluated. Endovascular treatment was performed via a single-sided femoral approach using a 4F introducer sheath, first with the coeliac axis (where possible), followed by the superior mesenteric artery (SMA). A trapping embolization technique was used (i.e. passage via aneurysm and embolization of the afferent and efferent arteries). A mixture of lipiodol and N-butyl cyanoacrylate was used for embolization (ratio, 1: 1). If this was not feasible, the aneurysm and the afferent artery were occluded with micro coils. Technical success was defined as the absence of any visible aneurysm in the completion angiography.

In patient undergoing surgery, the kocher maneuver was followed by the dissection of the adhesion between the pancreas' uncinate process and the aneurysm. The origin of the SMA was freed proximal to the aneurysm and the origin of the PDA was suspended. The distal part of the aneurysm was freed until the gastroduodenal artery and the aneurysm were isolated (Figure 1). After the aneurysmectomy, the vascular defect was repaired with the "shoemaker" technique using $6 / 0$ polypropylene suture (Figure 2). In order to prevent any future recurrence, a 2.5 $\mathrm{cm}$ wide and $5 \mathrm{~cm}$ long PTFE graft was wrapped around the PDA (Figure 3).

Statistical analysis was performed using the SPSS version 19 software program (IBM Corp., Armonk, NY, USA). The results were expressed as percentages and mean with minimum and maximum values.

\section{Results}

A total of 5 patients with PDA, of which 2 (40\%) were male, were evaluated. The mean age of the patients was 58.4 years (range, 16-82 years); the most common complaint was abdominal pain. All patients had a PDA aneurysm demonstrated by a computerized tomography (CT) or conventional angiography. The mean aneurysm size was $2.7 \mathrm{~cm}$ (range, $1.8-4.5 \mathrm{~cm}$ ). The mean duration of symptom onset was 35.6 days (range, 3-114 days). None of the patients had documented systemic vasculitis or coagulation disorder. The 16-year-old patient, who was the youngest, was being treated for Marfan syndrome. One patient was being followed-up for chronic pancreatitis since 2 years. One patient with coeliac axis occlusion had thrombus while 1 patient with coeliac axis stenosis had median arcuate ligament compression. In these patients the common hepatic artery filled via collateral circulation from the branches of SMA.
Possible risk factors for PDA aneurysm in patients were coeliac trunk stenosis due to arcuate ligament compression, coeliac trunk occlusion due to thrombus, hypertension, Marfan syndrome, and chronic pancreatitis.

Patient number one, 55-year-old male with right upper quadrant pain, had a fusiform aneurysm with $4.5 \mathrm{~cm}$ width caused by PDA (Figure 4). This patient underwent aneurysmectomy due to coeliac stenosis, collateral filling of the common hepatic artery, and an aneurysm size of 4.5 $\mathrm{cm}$. Patient number two, 74-year-old female patient had 2.2 $\mathrm{cm}$ PDA aneurysm which was discovered incidentally during a routine check-up. The patient, refused the recommended interventional treatment techniques, and was therefore prescribed with enoxaparin $40 \mathrm{mg} /$ day for 12 months for embolism prophylaxis. The patient did not suffer aneurysm expansion or any complication during a 2year follow-up. Patient number three, four and five were treated with embolization. Patient number three, a 16-yearold female patient who was being treated for Marfan syndrome had a $2.5 \mathrm{~cm}$ PDA aneurysm. Patient number four, an 84-year-old female being followed-up for chronic pancreatitis, presented at the hospital with sudden abdominal pain and following examination was found to have a $2.4 \mathrm{~cm}$ PDA aneurysm. An angiography confirmed the aneurysm had ruptured, and she was treated with endovascular embolization. Patient number five, a 65-yearold man, died due to acute myocardial infarction after the embolization of a $1.8-\mathrm{cm}$ aneurysm. Table 1 summarizes the clinical properties and treatment methods of the study patients.

\section{Discussion}

PDA aneurysms are pseudoaneurysms that form as a result of trauma, congenital malformations, pancreatitis, connective tissue disorders, or systemic vasculitis. True aneurysms are rarer and are reported to occur as a result of coeliac axis stenosis (6). The disorder may be asymptomatic, but may also manifest with a clinical presentation ranging from abdominal pain to hemorrhagic shock due to aneurysm rupture. One of our patients was diagnosed while she was in hemorrhagic shock due to PDA aneurysm rupture, while the other patients were diagnosed following chronic abdominal pain. Although sex predilection of the disease is not clear, men are reportedly more commonly affected (7). However, while PDA aneurysm rupture is reported among young, female patients with large aneurysms in Western Europe, it is more commonly reported among elderly men, and those with small aneurysms in Eastern Asia $(8,9)$. Two of our patients were male. Whereas none of our male patients had aneurysm rupture, the only patient with aneurysm rupture was a woman who was treated with embolization.

The exact mechanism of PDA aneurysm development has not been fully explained. It is considered that arterial hypertension and increased blood flow to small vessels result in true aneurysm development (10). In the majority of patients there exists coeliac axis stenosis, and 10-30\% of patients have median arcuate ligament compression (11). 
Table 1: Details of the patients treated for pancreaticoduodenal artery aneurysm.

\begin{tabular}{|lccccc|}
\hline Patient & $\mathbf{1}$ & $\mathbf{2}$ & $\mathbf{3}$ & $\mathbf{4}$ & $\mathbf{5}$ \\
\hline Age $(\mathbf{y}) /$ Sex & $55 / \mathrm{M}$ & $74 / \mathrm{F}$ & $16 / \mathrm{F}$ & $82 / \mathrm{F}$ & $65 / \mathrm{M}$ \\
\hline Etiology & $\begin{array}{c}\text { Coeliac } \\
\text { stenosis }\end{array}$ & $\begin{array}{c}\text { Coeliac } \\
\text { occlusion }\end{array}$ & $\begin{array}{c}\text { Marfan } \\
\text { syndrome }\end{array}$ & $\begin{array}{c}\text { Chronic } \\
\text { pancreatitis }\end{array}$ & Hypertension \\
\hline Size of aneurysm $(\mathbf{c m})$ & 4.5 & 2.2 & 2.5 & 2.4 & 1.8 \\
\hline MAL compression & $\mathrm{Y}$ & $\mathrm{N}$ & $\mathrm{N}$ & $\mathrm{N}$ & $\mathrm{N}$ \\
\hline Coeliac stenosis/occlusion & $\mathrm{Y}$ & $\mathrm{Y}$ & $\mathrm{N}$ & $\mathrm{N}$ & $\mathrm{N}$ \\
\hline Hepatic artery circulation & Collateral & Collateral & Coeliac & Coeliac & Coeliac \\
\hline Treatment & Surgery & LMWH & Embolization & Embolization & Embolization \\
\hline Follow up $(\mathbf{m o})$ & 6 & 10 & 10 & 14 & None \\
\hline Result & Favorable & Favorable & Favorable & Favorable & Exitus \\
\hline
\end{tabular}

$F$, female; LMWH, low molecular weight heparin; M, male; MAL: median arcuate ligament

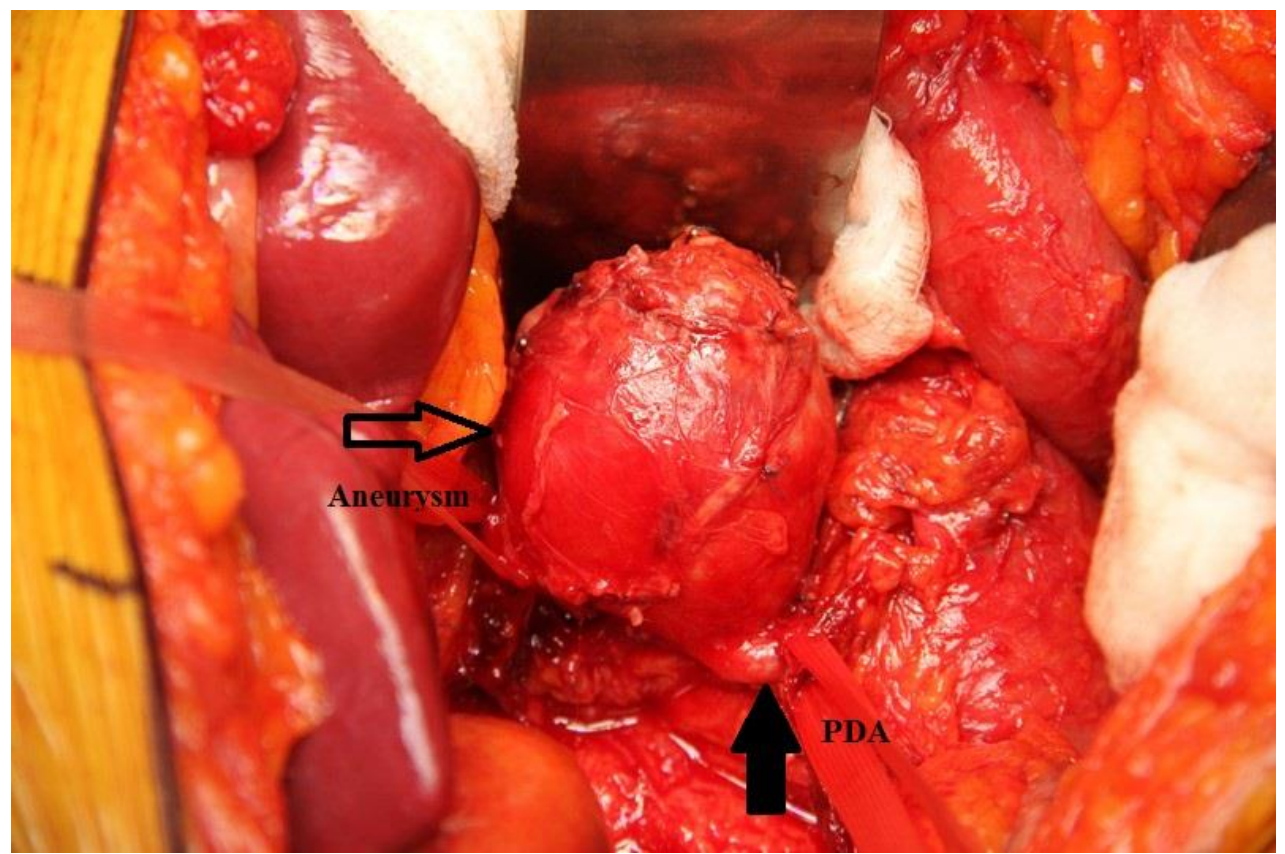

Figure 1: Dissected aneurysm. Black arrow shows the origin of PDA, the hollow arrow shows the aneurysm.

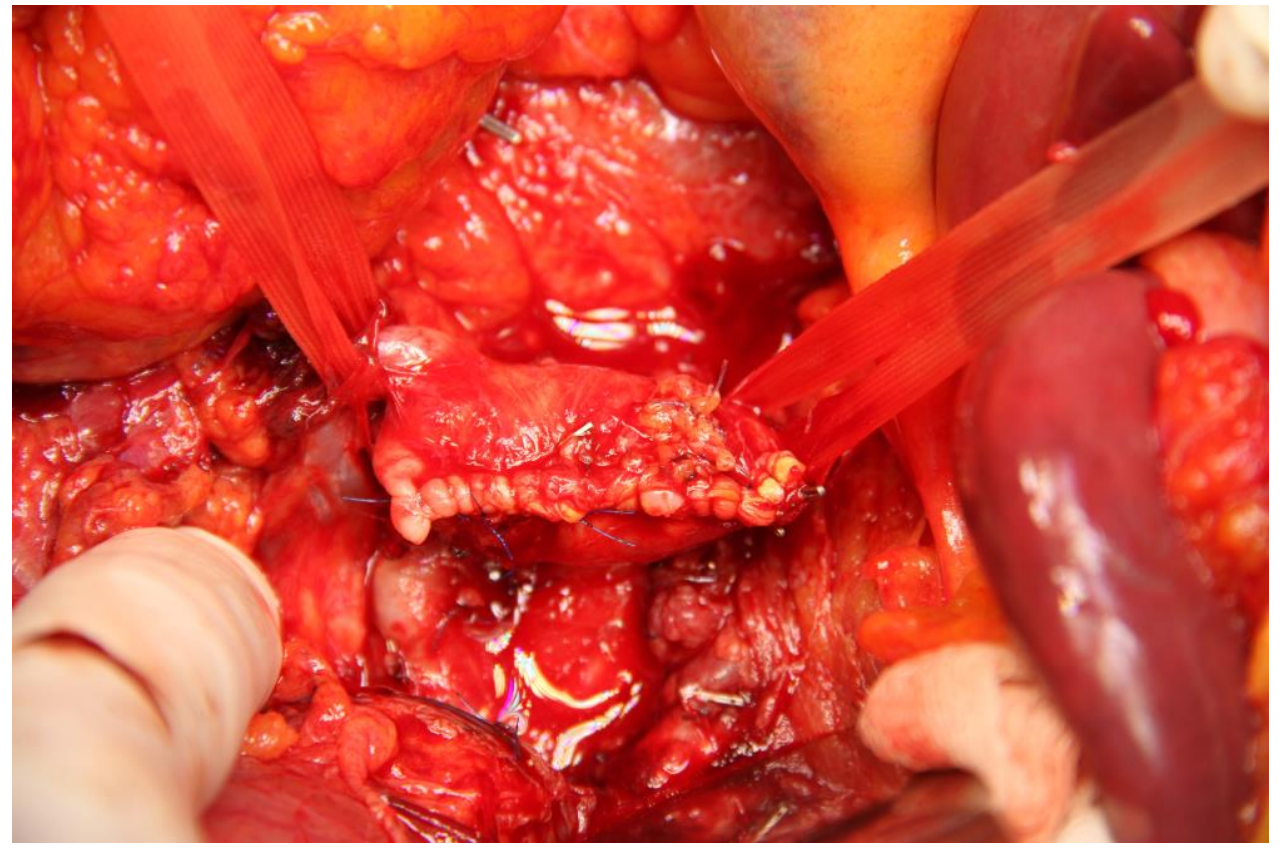

Figure 2: Repaired vascular defect. 


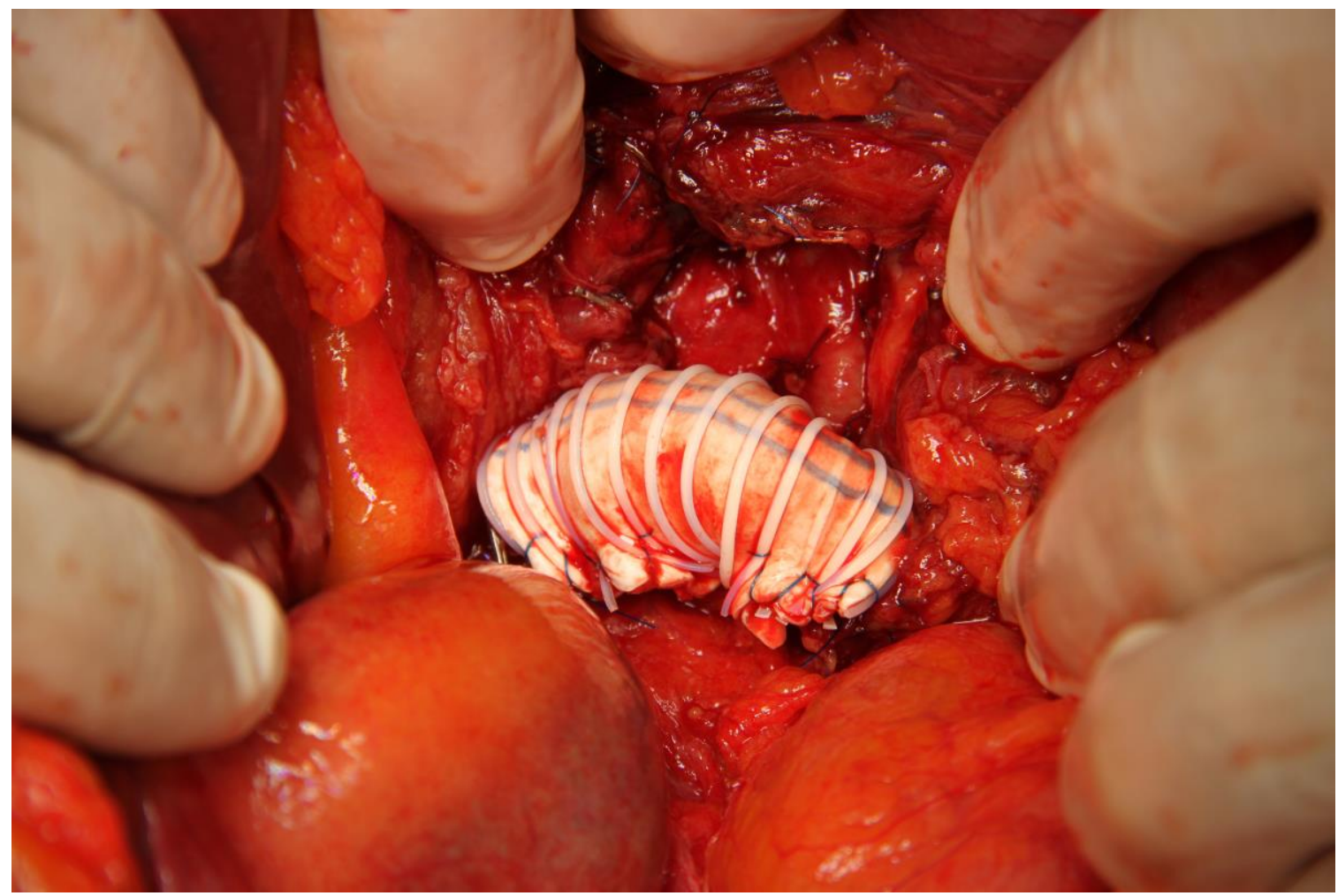

Figure 3: Supporting with PTFE graft.

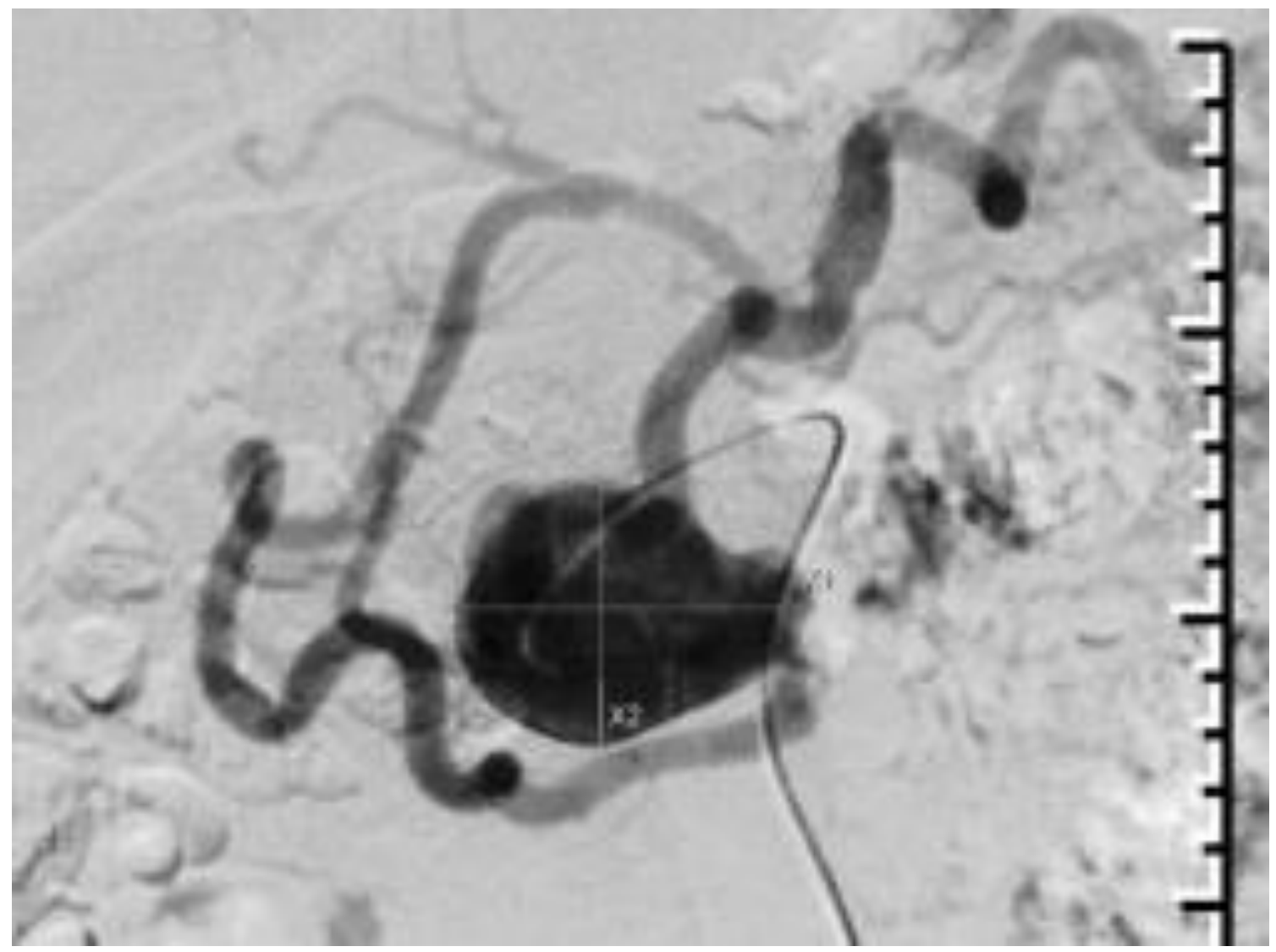

Figure 4: Angiography image of aneurysm 
We had 2 patients with coeliac stenosis or occlusion and one of them had median arcuate ligament compression.

Our youngest patient had Marfan syndrome. It is hypothesized that vascular disease in the Marfan syndrome occurs when FBN1 mutations prevent elastic fiber maturation by disrupting microfibrillar array. As a result, increase in inflammation and recurrent pressure creates weak regions in the adventitia layer, which is predisposed to aneurysm development (12). Since it has been demonstrated that PDA aneurysms may develop among patients without median arcuate ligament compression, abnormal coeliac axis flow, or hypertension at the pancreaticoduodenal arch, the pathophysiology of the disease has not been fully explained.

As for the treatment of the disease, trans-arterial embolization (TAE) is reported to confer a lower mortality compared to surgical therapy. The mortality rate among patients with a patent hepatic artery flow that undergo TAE has been reported as $0-2.1 \%$ (13). It was shown that PDA aneurysm rupture in patients with an aneurysm size below $10 \mathrm{~mm}$ can be safely treated with TAE and that the lipiodol mixture used for embolization is safe (11). One of our patients who had been treated with endovascular technique died due to myocardial infarction after the procedure. Coeliac devascularization is recommended during endovascular treatment of PDA aneurysm in patients with co-existing coeliac stenosis. This has been reported to lead to a reduction of arterial pressure on PDA and to reduce recurrence rates (14). Similar results have been reported for patients that did not undergo coeliac devascularization (15). In our patient group, on the other hand, coeliac devascularization was not applied and no additional problems were seen during follow-up.

Surgical treatment is applied for ruptured or large aneurysms for which endovascular intervention is not feasible. Surgical outcomes depend on surgical expertise and the patient's clinical condition. The mortality rate of surgical treatment has been reported between $13 \%$ and $20 \%$ (13). Since the aneurysm may be embedded in the deep pancreatic tissue in close to $70 \%$ of patients, the morbidity of surgical therapy is high (16). In our patient who was treated surgically, the aneurysm was embedded in the uncinate process of the pancreas. In this patient, surgery involved pancreatic tissue dissection followed by aneurysmectomy and supported with a PTFE graft; he developed no postoperative complications. Preservation of the native arterial tissue and external reinforcement was reported in 1982 by Egloff (17). It is reported that morbidity and mortality rates in this technique are comparable to open graft replacement (18). In addition, it has been argued in the literature that the wrapping technique is safer than conventional treatment methods when performed in suitable patients (19). In our patient, we preferred wrapping technique in order not to create hepatic artery ischemia with collateral filling from PDA. Apart from all these treatment options, conservative management has also been reported to cause no complications, as was the case for our medically-treated patient (14). It is recommended that the treatment method applied should be selected on the basis of the rupture rate of the aneurysm, mortality rate of the applied treatment, and patient age (20).

\section{Conclusion}

The rare nature of the disease, different etiological factors, and multiple vascular problems accompanying the disorder creates difficulties for establishing a treatment algorithm. In this study, treatment standardization could not be achieved due to the retrospective design of the study, and each patient having a different clinical presentation. Previous studies in the literature involve small numbers of patients as does our study. We observed no significant differences between our patients treated with different treatment modalities with respect to outcome and follow-up duration. Still, there is a need for prospective multi-center studies for establishing diagnostic and therapeutic algorithms.

\section{Acknowledgement: None}

Conflict of Interest: The authors declare no potential conflicts of interest with respect to the research, authorship, and/or publication of this article.

Author's Contributions: HOA, EHAS, FB, SY, MH; Planing of research, patient examination, surgical operations and collecting data: HOA; analyzing data, preparing the article and revisions.

Ethical issues: All Authors declare, Originality and ethical approval of research. Responsibilities of research, responsibilities against local ethics commission are under the Authors responsibilities.

\section{References}

1. Stanley JC, Wakefield TW, Graham LM, Whitehouse WM, Zelenock GB, Lindenauer S. Clinical importance and management of splanchnic artery aneurysms. Journal of Vascular Surgery. 1986;3:836-840. doi: 10.1016/0741-5214(86)90059-5.

2. Sutton D, Lawton G. Coeliac stenosis or occlusion with aneurysm of the collateral supply. Clinical Radiology. 1973;24:49-53.

3. Suzuki K, Kashimura H, Sato M, Hassan M, Yokota H, Nakahara A, Muto H, Yuzawa K, Fukao K, Tanaka N. Pancreaticoduodenal artery aneurysms associated with coeliac axis stenosis due to compression by median arcuate ligament and coeliac plexus. Journal of Gastroenterology. 1998;33:434-438.

4. Bracale G, Porcellini M, Bernardo B, Selvetella L, Renda A. Inferior pancreaticoduodenal artery aneurysm associated with common hepatic artery occlusion. The Journal of Cardiovascular Surgery. 1996;37:579-581.

5. Moore E, Matthews MR, Minion DJ, Quick R, Schwarcz TH, Loh FK, Endean ED. Surgical management of peripancreatic arterial aneurysms. Journal of Vascular Surgery. 2004;40(2):247-53. doi: 10.1016/j.jvs.2004.03.045

6. Armstrong MB, Stadtlander KS, Grove MK. Pancreaticoduodenal artery aneurysm associated with median arcuate ligament syndrome. Annals of Vascular Surgery. 2014;28(3):741-5. doi: 10.1016/j.avsg.2013.06.030.

7. Zelenock GB, Stanley JC. Splanchnic artery aneurysms. In: Rutherford RB, ed. Vascular Surgery. 5th ed. Philadelphia (PA): WB Saunders; 2000;1369-82. 
8. Flood K, Nicholson AA. Inferior pancreaticoduodenal artery aneurysms associated with occlusive lesions of the coeliac axis: diagnosis, treatment options, outcomes and review of the literature. Cardiovascular and Interventional Radiology. 2013;36:578-87. doi: 10.1007/s00270-012-0473-2.

9. Takase A, Akuzawa N, Hatori T, et al. Two patients with ruptured posterior inferior pancreaticoduodenal artery aneurysms associated with compression of the coeliac axis by the median arcuate ligament. Annals of Vascular Diseases. 2014;7:87-92. doi: 10.3400/avd.cr.1300105.

10. Kobayashi T, Uenoyama S, Isogai S. Successful transcatheter arterial embolization of an inferior pancreaticoduodenal artery aneurysm associated with coeliac axis stenosis. Journal of Gastroenterology and Hepatology. 2004;19:599-601. doi: 10.1111/j.14401746.2003.02894.x.

11. Chivot C, Rebibo L, Robert B, Regimbeau JM, Yzet T. Ruptured pancreaticoduodenal artery aneurysms associated with coeliac stenosis caused by the median arcuate ligament: a poorly known etiology of acute abdominal pain. European Journal of Vascular and Endovascular Surgery. 2016;51(2):295-301. doi: 10.1016/j.ejvs.2015.10.025.

12. Pereira L, Andrikopoulos K, Tian J, Lee SY, Keene DR, Reinhardt DP, Sakai LY, Biery NJ, Bunton T, Dietz HC, Ramirez F. Targetting of the gene encoding fibrillin-1 recapitulates the vascular aspect of Marfan syndrome. Nature Genetics. 1997;17(2):218-222. doi: 10.1038/ng1097-218.

13. Coll DP, Ierardi RT, Kerstein MD, Yost S, Wilson A, Matsumoto T. Aneurysms of the pancreaticoduodenal arteries: a change in management. Annals of Vascular Surgery. 1998;22:286-291. doi: http://dx.doi.org/10.1007/s100169900155.
14. Tien YW, Kao HL,Wang HP. Coeliac artery stenting: a new strategy for patients with pancreaticoduodenal artery aneurysm associated with stenosis of the coeliac artery. Journal of Gastroenterology. 2004;39:81-85. doi: 10.1007/s00535-003-1251-3.

15. Sgroi MD, Kabutey NK, Krishnam M, Fujitani RM. Pancreaticoduodenal artery aneurysms secondary to median arcuate ligament syndrome may not need coeliac artery revascularization or ligament release. Annals of Vascular Surgery. 2015;29(1):122.e1-7. doi: 10.1016/j.avsg.2014.05.020

16. Retzlaff JA, Hagedom AB, Bartholomew LG. Abdominal exploration for gastrointestinal bleeding of obscure origin. JAMA. 1961;177:104-7.

17. Egloff L, Rothlin M, Kugelmeier J, Senning A, Turina M. The ascending aortic aneurysm: replacement or repair? The Annals of Thoracic Surgery. 1982;34:117-24. doi: 10.1016/S00034975(10)60871-5.

18. Plonek T. A meta-analysis and systematic review of wrapping of the ascending aorta. Journal of Cardiac Surgery. 2014;29:809-15. doi: $10.1111 /$ jocs. 12448 .

19. Pecoraro F, Shingaki M, Steuer J, Chaykovska L, Rancic Z, Weber A, Nguyen-Kim TD, Bettex D, Veith FJ, Lachat M. Treatment of isolated ascending aortic aneurysm by off-pump epiaortic wrapping is safe and durable. Interactive CardioVascular and Thoracic Surgery. 2016;23(1):286-291. doi: 10.1093/icvts/ivw103.

20. Takao H, Nojo T, Ohtomo K. True pancreaticoduodenal artery aneurysms: A decision analysis. European Journal of Radiology. 2010;75:110-113. doi: 10.1016/j.ejrad.2009.03.041.

Copyright (C) 2018 The Author(s); This is an open-access article distributed under the terms of the Creative Commons Attribution License (http://creativecommons.org/licenses/by/4.0), which permits unrestricted use, distribution, and reproduction in any medium, provided the original work is properly cited. All Rights reserved by international journal of Medical Science and Discovery. 